\title{
Caracterização e distribuição das formas do nitrogênio orgânico em três solos da Amazônia Central
}

\author{
S. S. ALFAIA ${ }^{1}$
}

\section{RESUMO}

As formas orgânicas do nitrogênio em solos são determinadas mediante a identificação e quantificação dos compostos orgânicos liberados, quando os solos são tratados com ácido a alta temperatura. Ainda não se conhecem na literatura trabalhos sobre a natureza química do $\mathrm{N}$ orgânico nos solos da Amazônia. O presente trabalho teve como objetivos identificar e quantificar a transformação do nitrogênio proveniente de fertilizantes marcados com ${ }^{15} \mathrm{~N}$ nas frações orgânicas nitrogenadas de três solos da Amazônia Central: dois solos de terra firme, classificados como Latossolo Amarelo e Podzólico Vermelho Amarelo e um solo de várzea, classificado como Glei Pouco Húmico. Foram utilizadas amostras de solos de um ensaio de adubação desenvolvido em condições de casa de vegetação, onde, após cultivo, procedeu-se o fracionamento do $\mathrm{N}$ orgânico do solo por meio da hidrólise ácida. Foram determinadas as seguintes frações: N-solúvel em ácido e destilado (NSAD), N-solúvel em ácido e não destilado (NSAnD) e N-não hidrolisado (NnH). Nos solos de terra firme, o $\mathrm{N}$ orgânico foi encontrado principalmente na forma de $\mathrm{N}$ solúvel em ácido e não destilado (NSAnD). Entre 63 a 66\% (Latossolo) e 69 a 73\% (podzólico) do ${ }^{15} \mathrm{~N}$ imobilizado no solo foram encontrados na fração NSAnD. Esses resultados demonstram a importância da imobilização microbiana do N nesses solos. No solo de várzea, ao contrário, houve pouca diferença entre os teores de ${ }^{15} \mathrm{~N}$ do fertilizante imobilizado nas frações NSAnD e NSAD. Entre 46 e $53 \%$ do total de ${ }^{15} \mathrm{~N}$ imobilizado foram encontrados na fração NSAnD, enquanto que 42 a 52\% ficaram na fração NSAD. Nesse solo, a presença de argila tipo 2:1 pode ter contribuído para o alto estoque de ${ }^{15} \mathrm{~N}$ orgânico incorporado na fração $\mathrm{NSAD}$, devido à fixação de íons $\mathrm{NH}_{4}^{+}$.

\section{PALAVRAS-CHAVE}

imobilização, fracionamento do N-orgânico do solo, hidrólise ácida, fertilizantes nitrogenados.

\section{Characterization and distribution of organic forms of nitrogen in three soils of Central Amazônia}

\begin{abstract}
The organic $N$ forms in soil are determined by identification and quantification of organic compounds released by acid hydrolysis. There are no available data on the chemical nature of organic $N$ in the Amazon soils. The objective of this study was to identify and quantify the transformation of ${ }^{15} \mathrm{~N}$-labeled nitrogen fertilizers to different organic nitrogen fractions in three soils of Central Amazonia: an Oxisol, an Ultisol and a Low-Humic Gley (LHG). The soils were sampled after cultivation in greenhouse experiment. The chemical fractionation of soil organic nitrogen, as acid-hydrolysable and distillable N(NSAD), acid-hydrolysable non-distillable N(NSAnD) and non-hydrolysable $N$ $(\mathrm{NnH})$, was performed. In the Oxisol and Ultisol, 63 to $66 \%$ and 69 to $73 \%$, respectively, offertilizer N applied was incorporated in the $N S A n D$ fraction. This suggests that $N$ immobilization of microbial origin is important in these soils. In the LHG, there was equal immobilization in both NSAnD and NSAD fractions: 46 to 53\% of N applied was immobilized in NSAnD, while 42 to $53 \%$ was immobilized in NSAD. In this soil, the presence of 2:1 type clays probably contributed to the high stock of ${ }^{15} N$ incorporated in the NSAD fraction, due to fixation of $\mathrm{NH}_{4}^{+}$.
\end{abstract}

\section{KEY WORDS}

immobilization, fractionation of organic- $N$, acid hydrolysis, nitrogen fertilizers. 


\section{INTRODUÇÃO}

O nitrogênio encontra-se no solo essencialmente na forma orgânica (aproximadamente 98\%). A outra pequena parte encontra-se nas formas minerais de amônio, nitrato e nitrito. A mineralização é a transformação biológica do $\mathrm{N}$ orgânico do solo em N inorgânico, executada pelos microrganismos heterotróficos do solo. A imobilização refere-se ao processo inverso, ou seja, é a transformação do N inorgânico em orgânico. Os microrganismos do solo assimilam as formas inorgânicas de $\mathrm{N}$ para formar os constituintes orgânicos de suas células e tecidos. Os compostos sintetizados pelos microrganismos podem ser parcialmente mineralizados e tornar-se disponível para as plantas. Estudos sobre os mecanismos relacionados com a imobilização e mineralização de fertilizantes nitrogenados têm sido relatados por diversos autores (Broadbend \& Taylor, 1962; Vong et al., 1990, Jacquin et al., 1992, Sulce et al., 1996).

Como consequiência dos processos de mineralização e imobilização, uma parte não negligenciável (20 a 50\%) do N dos fertilizantes aplicada ao solo é imobilizada e incorporada nas estruturas das substâncias húmicas, tornando-se pouco disponível para as plantas (Jacquin et al., 1992). Os experimentos com ${ }^{15} \mathrm{~N}$ têm mostrado que aproximadamente $1 / 3$ do $\mathrm{N}$ dos fertilizantes aplicados permanece no solo na forma orgânica após o primeiro cultivo, e somente uma pequena fração $(<15 \%)$ é aproveitada pela planta em um cultivo subseqüente (Kelley $\&$ Stevenson, 1995).

As formas orgânicas do nitrogênio em solos são determinadas mediante a identificação e quantificação dos compostos orgânicos liberados, quando os solos são tratados com ácido a alta temperatura. Qualitativamente se podem distinguir as seguintes formas de $\mathrm{N}$ orgânico do solo: 1) $\mathrm{N}$ solúvel em ácido e não destilável (NSAnD), fração constituída essencialmente pelos aminoácidos, representa o compartimento biológico onde se estabelecem os processos de imobilização e mineralização (Vong, 1987); 2) N solúvel em ácido e destilável (NSAD), forma de $\mathrm{N}$ essencialmente de natureza amoniacal e 3) $\mathrm{N}$ não hidrolisável $(\mathrm{NnH})$, que corresponde a fração do $\mathrm{N}$ resistente à hidrólise ácida de natureza não protéica (Schnitzer, 1981). Ainda não se conhecem na literatura trabalhos sobre a natureza química do $\mathrm{N}$ orgânico nos solos da Amazônia. Somente com a utilização de ${ }^{15} \mathrm{~N}$ é possível avaliar as transformaçōes que sofre o $\mathrm{N}$ dos fertilizantes no solo, além de possibilitar uma melhor caracterização das frações do $\mathrm{N}$ orgânico que são susceptíveis a mineralização microbiana, em formas aproveitáveis pelas plantas.

O presente trabalho teve como objetivo caracterizar e avaliar a dinâmica da transformação do nitrogênio dos fertilizantes marcados com ${ }^{15} \mathrm{~N}$, nas fraçōes orgânicas nitrogenadas em três solos da Amazônia Central.

\section{MATERIAL E MÉTODOS}

Foram utilizadas amostras coletadas na profundidade de 0 $30 \mathrm{~cm}$ de dois solos de terra firme da região de Manaus, classificados como Latossolo Amarelo textura muito argilosa e Podzólico Vermelho Amarelo textura areno/argilosa, e de um solo de várzea classificado como Glei Pouco Húmico $(\mathrm{GPH})$ do Careiro, localizado em uma ilha situada após a confluência do rio Solimōes e Rio Negro na região da Amazônia Central. As principais propriedades químicas e a granulometria desses solos são: a) Latossolo - pH em água, 4,6; $\mathrm{N}$ total, $1,4 \mathrm{~g} \mathrm{~kg}^{-1}$; $, 17,8 \mathrm{~g} \mathrm{~kg}^{-1}$; CTC, 54 mmolc kg-1 e, 110, 50 e $840 \mathrm{~g} \mathrm{~kg}^{-1}$ de areia, silte e argila, respectivamente; b) podzólico - $\mathrm{pH}$ em água, 4,7; $\mathrm{N}$ total, $0,51 \mathrm{~g} \mathrm{~kg}^{-1}$; C, 10,9 $\mathrm{g} \mathrm{kg}^{-1}$; CTC, 24 mmolc kg-1 e, 640, $60 \mathrm{e}$ $300 \mathrm{~g} \mathrm{~kg}^{-1}$ de areia, silte e argila, respectivamente; c) GPH - pH em água, 6,4; $\mathrm{N}$ total, $0,73 \mathrm{~g} \mathrm{~kg}^{-1}$; C, 8,9 $\mathrm{g} \mathrm{kg}^{-1}$; CTC, 96 mmolc kg-1 e 500, 390 e $110 \mathrm{~g} \mathrm{~kg}^{-1}$ de areia, silte e argila, respectivamente. $\mathrm{O} \mathrm{C} \mathrm{e} \mathrm{o} \mathrm{N}$ foram determinados por cromatografia gasosa, utilizando um auto-analisador $\mathrm{CHN}$ (Carlo-Erba 500), o pH, a CTC e a granulometria foram determinados segundo a metodologia da EMBRAPA (1997).

As amostras de solos utilizadas nesse estudo foram provenientes de um ensaio de adubação desenvolvido em condiçôes de casa de vegetação, submetidos a três tratamentos, constituídos pela testemunha (sem aplicação de N), e $60 \mathrm{mg} \mathrm{kg}^{-1} \mathrm{de} \mathrm{N}$ na forma de uréia e de sulfato de amônio, respectivamente com 34,5 e $40 \%$ de átomos em excesso de ${ }^{15} \mathrm{~N}$. Em seguida esses solos foram cultivados com azevém da Itália (Lolium multiflorum L.), por um período de 63 dias. O delineamento experimental foi de blocos casualizados com quatro repetições, sendo que após o cultivo tomaram-se duas repetiçōes de cada tratamento e procedeu-se o fracionamento do $\mathrm{N}$ orgânico do solo. A significação dos dados foi determinada pela análise de variância e teste de Tukey a 5\% de probabilidade. Neste trabalho são abordados apenas os resultados do fracionamento, uma vez que os dados referentes ao balanço do ${ }^{15} \mathrm{~N}$ no sistema solo-planta, foram estudados por Alfaia $(1995 ;$ 1997).

O método de determinação das frações orgânicas de nitrogênio correspondeu a uma técnica simplificada do processo proposto por Stewart et al. (1963). O equivalente a $10 \mathrm{~g}$ de solo seco (após extração do $\mathrm{N}$ mineral com $\mathrm{KCl} 1 \mathrm{M}$ ) foi submetido a extração sob refluxo, com $100 \mathrm{ml}$ de $\mathrm{HCl} 6 \mathrm{M}$ durante 16 horas (Bremner, 1965). Após resfriamento, a solução do hidrolisado foi filtrada e o solo residual foi seco ao ar. Nesse solo, o $\mathrm{N}$ que corresponde à fração de $\mathrm{N}$-não hidrolisado $(\mathrm{NnH})$, foi determinado pelo processo de digestão ácida a quente Por destilação de uma alíquota da solução hidrolisada na presença de $\mathrm{NaOH} 10 \mathrm{M}$, obteve-se a fração do $\mathrm{N}$-solúvel em ácido e destilado (NSAD). Pela digestão ácida a quente do hidrolisado obteve-se a fração correspondente ao $\mathrm{N}$-hidrolisado total. $\mathrm{O}$ valor de $\mathrm{N}$ solúvel em ácido e não destilado (NSAnD) foi obtido pela 
diferença entre o N-hidrolisado total e o NSAD. As análises isotópicas de ${ }^{15} \mathrm{~N}$ das amostras de solos foram efetuadas por espectrometria de massa (Fiedler \& Proksch, 1975).

\section{RESULTADOS E DISCUSSÃO}

Nos três solos observa-se que o $\mathrm{N}$ orgânico encontra-se principalmente na forma de NSAnD (Tabela 1), cujos teores foram significativamente mais elevados do que os observados nas outras formas. O teor de NSAD variou entre 24,2 e $25,7 \%$ no Latossolo e de 21,2 a 27,6\% no Podzólico, enquanto que no GPH os valores foram um pouco mais elevados e situou-se entre 29,3 a 33,2\%. Essa forma de $\mathrm{N}$ orgânico de natureza amoniacal representa, normalmente, de 18 a 32\% do $\mathrm{N}$ total (Schnitzer, 1981). O teor de $\mathrm{NnH}$ variou entre 22,3 e $26,7 \%$ no Latossolo, 12,2 a $15,4 \%$ no Podzolico e de 24,3 a 26,2\% no GPH. Essa fração corresponde teoricamente ao $\mathrm{N}$ heterocíclico resistente à hidrólise ácida, sendo a fração mais estável das substâncias húmicas presentes no solo. Segundo Kelley \& Stevenson (1995), esta fração representa cerca de 25 a 35\% do N total do solo.

Existem grandes variações na composição do $\mathrm{N}$ orgânico nos solos. Essas variações normalmente têm sido atribuídas às propriedades físicas, químicas e biológicas do solo. Nos três solos estudados o teor de $\mathrm{N}$ orgânico foi significativamente mais elevado no Latossolo, seguido do GPH e Podzólico.

\section{DISTRIBUIÇÃO DAS DIFERENTES FORMAS ORGÂNICAS DO ${ }^{15} \mathrm{~N}$ DO FERTILIZANTE IMOBILIZADO}

$\mathrm{O}{ }^{15} \mathrm{~N}$ dos fertilizantes imobilizado no solo após o cultivo foi determinado sem considerar o $\mathrm{N}$ mineral restante no solo, devido

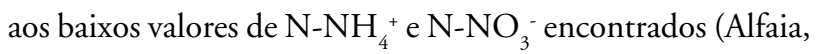
1997), mostrando que durante o seu desenvolvimento a planta absorveu o $\mathrm{N}$ mineral disponível no solo. No entanto, o $\mathrm{N}$ do fertilizante imobilizado no solo variou em função do tipo de solo e da forma do adubo aplicado. Nos Podzólico e Latossolo a imobilização foi significativamente mais elevada na presença de uréia do que na de sulfato de amônio, variando de 10,1 a 15,4\% no Podzólico e de 13,0 a 16,2\% no Latossolo (Figura 1). No GPH a diferença entre os dois fertilizantes foi menor e variou de 19,4 a 19,6\%. A importância dessa imobilização é que esta representa uma forma de estoque de $\mathrm{N}$ no solo relativamente estável, podendo contribuir para a manutenção desse nutriente

Tabela 1 - Teor de nitrogênio orgânico e distribuição das formas orgânicas de $\mathrm{N}$ em três solos da Amazônia Central.

\begin{tabular}{|c|c|c|c|c|}
\hline \multirow{2}{*}{ Tratamento } & \multirow{2}{*}{ N orgânico } & \multicolumn{3}{|c|}{ Formas orgânicas de $\mathrm{N}$} \\
\hline & & NSAD & NSAnD & $\mathrm{NnH}$ \\
\hline & $\mathrm{mg} \mathrm{kg}^{-1}$ & \multicolumn{3}{|c|}{ - . \% do N orgânico - . . - } \\
\hline & \multicolumn{4}{|c|}{ Latossolo } \\
\hline Testemunha & 1.549 & 25,6 & 47,8 & 26,7 \\
\hline$\left(\mathrm{NH}_{4}\right)_{2} \mathrm{SO}_{4}$ & 1.562 & 25,7 & 52,2 & 22,3 \\
\hline \multirow[t]{3}{*}{ Uréia } & 1.543 & 24,2 & 52,1 & 23,6 \\
\hline & $1.551 \mathrm{~A}$ & $25,2 \mathrm{~b}$ & 50,7 a & $24,2 \mathrm{~b}$ \\
\hline & \multicolumn{4}{|c|}{ Podzólico } \\
\hline Testemunha & 650 & 21,2 & 63,5 & 15,4 \\
\hline$\left(\mathrm{NH}_{4}\right)_{2} \mathrm{SO}_{4}$ & 679 & 22,5 & 62,4 & 15,1 \\
\hline \multirow[t]{3}{*}{ Uréia } & 603 & 27,6 & 60,2 & 12,2 \\
\hline & $644 \mathrm{C}$ & $23,8 \mathrm{~b}$ & $62,0 \mathrm{a}$ & $14,2 \mathrm{c}$ \\
\hline & \multicolumn{4}{|c|}{ Glei Pouco Húmico } \\
\hline Testemunha & 697 & 32,2 & 41,6 & 26,2 \\
\hline$\left(\mathrm{NH}_{4}\right)_{2} \mathrm{SO}_{4}$ & 729 & 33,2 & 44,8 & 25,0 \\
\hline \multirow[t]{2}{*}{ Uréia } & 719 & 29,3 & 46,4 & 24,3 \\
\hline & $715 B$ & $31,6 \mathrm{~b}$ & $44,3 \mathrm{a}$ & $25,2 \mathrm{c}$ \\
\hline
\end{tabular}

Médias seguidas por letras maiúsculas distintas nas colunas, e letras minúsculas distintas nas linhas, diferem entre si, ao nível de $5 \%$ de probabilidade, pelo teste de Tukey. NSAD (N-solúvel em ácido e destilado)

NSAnD (N-solúvel em ácido e não destilado) $\mathrm{NnH}$ (N-não hidrolisado)
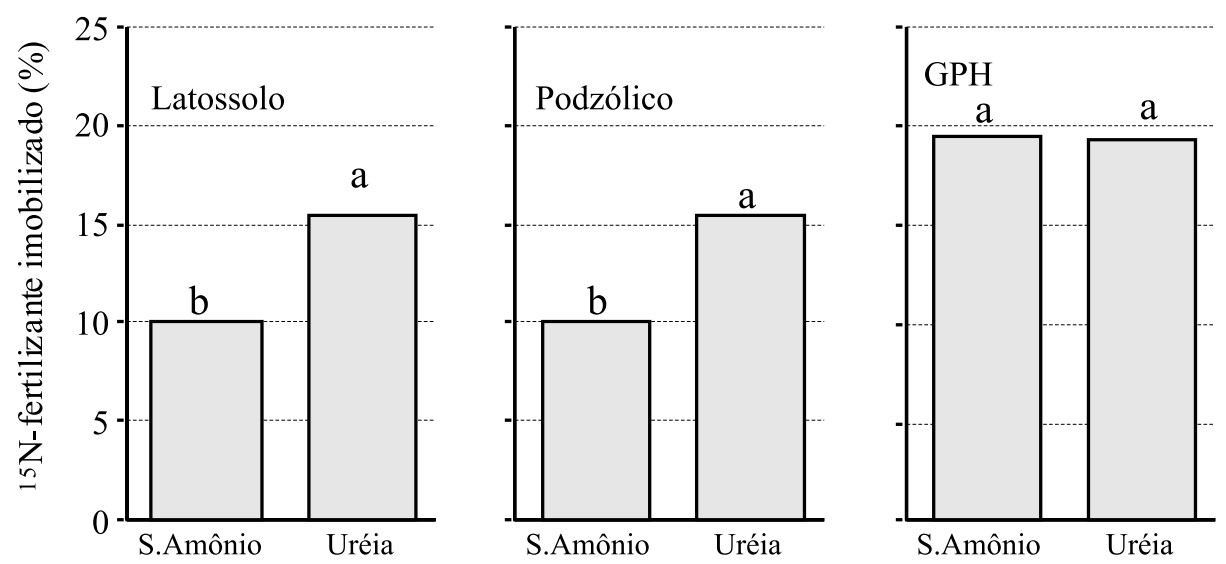

Figura 1 - Porcentagem de ${ }^{15} \mathrm{~N}$-fertilizante (uréia e sulfato de amônio) imobilizado no solo, após o cultivo do azevém em vasos em três solos da Amazônia Central. Letras diferentes indicam diferenças significativas ao nível de $5 \%$ de probabilidade pelo teste de Tukey. 
no solo em longo prazo (Jacquin \& Vong, 1989). O conhecimento das formas orgânicas nitrogenadas e análise de sua estabilidade podem-se constituir em um critério útil de avaliação do $\mathrm{N}$ orgânico do solo que é susceptível a mineralização (Egoumenides, et al., 1987).

Os valores da distribuição do $\mathrm{N}$ orgânico do fertilizante, imobilizado no solo, nas três fraçôes estudadas não apresentaram grandes variaçōes em relação aos tratamentos (Tabela 2). Alguns trabalhos demonstraram que a aplicação de fertilizante por um longo tempo não afetou significativamente a distribuição das formas de N no solo (Khan, 1971; Giddens et al., 1971). No Latossolo, a distribuição do ${ }^{15} \mathrm{~N}$ do fertilizante nas diferentes fraçōes orgânicas de N é semelhante à observada no Podzólico, para as duas formas de fertilizantes aplicados. Nesses solos houve um importante estoque de ${ }^{15} \mathrm{~N}$-fertilizante na fração $\mathrm{NSAnD}$ para ambas as formas de fertilizantes, enquanto que no GPH houve pouca diferença entre os teores de ${ }^{15} \mathrm{~N}$ imobilizado nas fraçoes NSAnD e NSAD. Por outro lado, nos três solos estudados foram observados baixos valores de ${ }^{15} \mathrm{~N}$ do fertilizante imobilizados na fração mais estável $(\mathrm{NnH})$.

A Figura 2 mostra que no Latossolo, 63\% (uréia) a 66\% (sulfato de amônio) do total de ${ }^{15} \mathrm{~N}$ imobilizado no solo foi encontrado na fração NSAnD. No Podzólico, 69\% (uréia) a $73 \%$ (sulfato de amônio) do total de ${ }^{15} \mathrm{~N}$ imobilizado, também ficou retido na fração NSAnD. Esses resultados demonstram a importância da imobilização de origem microbiana do $\mathrm{N}$ nesses solos. Jacquin et al., (1992) observaram que a fração NSAnD foi a forma predominante de ${ }^{15} \mathrm{~N}$ imobilizado, sendo também a mais alterada pelo cultivo e apresentando-se como a mais rapidamente disponível às plantas. Vong et al. (1990)

\begin{tabular}{|c|c|c|c|c|c|}
\hline Tratamentos & $\begin{array}{l}\text { Formas orgânicas } \\
\text { de } \mathrm{N}\end{array}$ & $\begin{array}{l}\text { Concentração } \\
\text { de N no solo }\end{array}$ & $\begin{array}{l}\text { Átomos de } \\
{ }^{15} \mathrm{~N} \text {-excesso }\end{array}$ & $\mathbf{Q}^{15} \mathbf{N}^{(2)}$ & 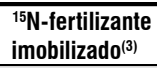 \\
\hline & & $\mathrm{mg} \mathrm{kg}^{-1}$ & $\%$ & $\mathrm{mg} \mathrm{kg}^{-1}$ & $\%$ \\
\hline & & \multicolumn{4}{|c|}{ Latossolo } \\
\hline \multirow{4}{*}{$\left(\mathrm{NH}_{4}\right)_{2} \mathrm{SO}_{4}$} & NSAD & 401 & 0,206 & 0,82 & 3,4 \\
\hline & NSAnD & 812 & 0,253 & 2,05 & 8,6 \\
\hline & $\mathrm{NnH}$ & 349 & 0,070 & 0,24 & 1,0 \\
\hline & N orgânico total & & - & 3,12 & 13,0 \\
\hline \multirow{4}{*}{ Uréia } & NSAD & 374 & 0,249 & 0,93 & 4,5 \\
\hline & NSAnD & 805 & 0,266 & 2,14 & 10,3 \\
\hline & $\mathrm{NnH}$ & 365 & 0,089 & 0,32 & 1,6 \\
\hline & N orgânico total & & - & 3,39 & 16,4 \\
\hline & & \multicolumn{4}{|c|}{ Podzólico } \\
\hline \multirow{4}{*}{$\left(\mathrm{NH}_{4}\right)_{2} \mathrm{SO}_{4}$} & NSAD & 153 & 0,393 & 0,60 & 2,5 \\
\hline & NSAnD & 424 & 0,415 & 1,76 & 7,3 \\
\hline & $\mathrm{NnH}$ & 103 & 0,060 & 0,06 & 0,3 \\
\hline & N orgânico total & & - & 2,42 & 10,1 \\
\hline \multirow{4}{*}{ Uréia } & NSAD & 166 & 0,549 & 0,911 & 4,4 \\
\hline & NSAnD & 363 & 0,598 & 2,171 & 10,5 \\
\hline & $\mathrm{NnH}$ & 74 & 0,109 & 0,080 & 0,4 \\
\hline & N orgânico total & & - & 2,281 & 15,3 \\
\hline & & \multicolumn{4}{|c|}{ Glei Pouco Húmico } \\
\hline \multirow{4}{*}{$\left(\mathrm{NH}_{4}\right)_{2} \mathrm{SO}_{4}$} & NSAD & 247 & 0,989 & 2,44 & 10,2 \\
\hline & NSAnD & 296 & 0,725 & 2,15 & 8,9 \\
\hline & $\mathrm{NnH}$ & 186 & 0,061 & 0,11 & 0,5 \\
\hline & $\mathrm{N}$ orgânico total & & - & 4,70 & 19,6 \\
\hline \multirow{4}{*}{ Uréia } & NSAD & 211 & 0,804 & 1,69 & 8,2 \\
\hline & NSAnD & 334 & 0,644 & 2,15 & 10,4 \\
\hline & $\mathrm{NnH}$ & 175 & 0,105 & 0,18 & 0,9 \\
\hline & $\mathrm{N}$ orgânico total & & - & 2,29 & 19,4 \\
\hline
\end{tabular}

(1) Aplicados $60 \mathrm{mg} \mathrm{kg}^{-1}$ de $\mathrm{N}$ na forma de uréia e sulfato de amônio, marcados respectivamente com $34,5 \%$ e $40 \%$ de átomos de excesso de ${ }^{15} \mathrm{~N}$. (2) ${ }^{15} \mathrm{~N}=\left(\mathrm{N}\right.$ orgânico xátomos \% de ${ }^{15} \mathrm{~N}$ em excesso na amostra) $/ 100$, onde $Q^{15} \mathrm{~N}$ é a quantidade de ${ }^{15} \mathrm{~N}$ na amostra. ${ }^{3)} \mathrm{N}$ proveniente do fertilizante $=\left(Q^{15} \mathrm{~N} / \mathrm{Q}^{15} \mathrm{~N}\right.$ do fertilizante $) \times 100$. 
encontraram correlação altamente significativa entre a fração NSAnD e a biomassa microbiana do solo, sendo que no solo cultivado um aumento mais acentuado da atividade biológica foi observado, induzindo uma maior porcentagem da fração NSAnD presente no solo. Sulçe et al. (1996) também mostraram a fração NSAnD como sendo a mais ativa do solo. Entre 26 a $27 \%$ do total de ${ }^{15} \mathrm{~N}$ imobilizado no Latossolo foi encontrado na fração NSAD, enquanto que no Podzólico, a taxa de imobilização
NSAnD, a porcentagem de ${ }^{15} \mathrm{~N}$ presente na fração NSAD decresceu muito pouco durante o cultivo. Por outro lado, Vong et al. (1990), relataram que nenhuma correlação significativa foi observada entre a fração NSAD e a biomassa microbiana do solo.

$\mathrm{O}$ teor de ${ }^{15} \mathrm{~N}$ imobilizado no solo na fração $\mathrm{NnH}$ para os três solos foi baixo, variando de 8 a 10\% no Latossolo, 2 a $5 \%$ no GPH, e permaneceu em torno de 2,5 \% no Podzólico, para ambas as formas de fertilizantes.

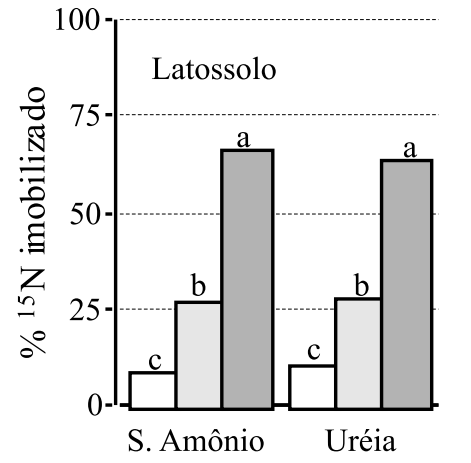

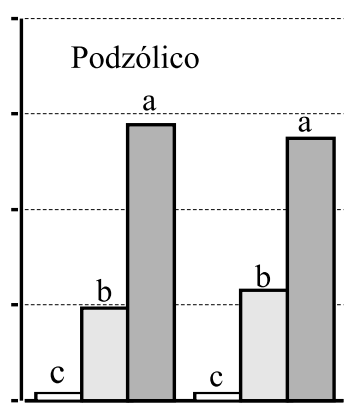

S. Amônio Uréia

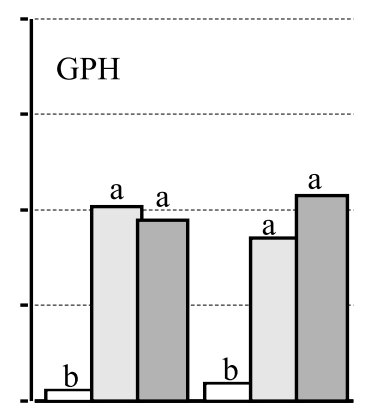

S. Amônio Uréia

$\mathrm{NnH}$

NSAD

NSAnD

Figura 2 - Porcentagem de ${ }^{15} \mathrm{~N}$-fertilizante imobilizado nas frações orgânicas nitrogenadas em três solos da Amazônia Central. NnH (N-não hidrolisado), NSAD (N-solúvel em ácido e destilado) e NSAnD (N-solúvel em ácido e não destilado). Letras diferentes indicam diferenças significativas ao nível de $5 \%$ de probabilidade pelo teste de Tukey.

nessa fração variou de 25 a 29\%. De acordo com Vong (1987), essa fração de natureza amoniacal não apresenta contribuição importante em relação ao estoque e transformação de $\mathrm{N}$ de origem microbiana. Tal fração obtida por destilação direta, é muito lábil e seus teores apresentam pequena variação ao longo do tempo. Segundo o referido autor, a fração NSAD corresponderia a formas de $\mathrm{N}$ de transformação muito rápida e representaria um ponto de passagem para outras formas de $\mathrm{N}$.

A figura 2 mostra que do total de ${ }^{15} \mathrm{~N}$ imobilizado no GPH, entre 46 e 53\% foram encontrados na fração NSAnD, nos tratamentos com sulfato de amônio e uréia, respectivamente, enquanto que $42 \%$ (uréia) a $52 \%$ (sulfato de amônio) do total de ${ }^{15} \mathrm{~N}$ imobilizado ficou na fração NSAD. Nesse solo a presença de argila tipo 2:1 pode ter contribuído para o alto estoque de ${ }^{15} \mathrm{~N}$ imobilizado na fração NSAD, devido à fixação de $\mathrm{NH}_{4}^{+}$(Alfaia \& Nogueira, 1985). Segundo Kelley \& Stevenson (1995), os ions amônio fixados nas argilas podem contribuir em grande parte para a elevação dos teores de N-orgânico na forma amoniacal. Os compostos nitrogenados retidos nas estruturas de latice dos minerais de argila podem ser particularmente resistentes ao ataque de microrganismos. Em um estudo sobre a mineralização do ${ }^{15} \mathrm{~N}$ do fertilizante imobilizado nas diversas fraçôes orgânicas do solo, Jacquin et al. (1992), observaram que ao contrário da fração

\section{CONCLUSÕES}

Os resultados mostraram que a taxa de imobilização do $\mathrm{N}$ do fertilizantes no solo variou em função do tipo de solo e da forma do adubo aplicado. A determinação da forma orgânica do $\mathrm{N}$ do fertilizante imoblizado mostrou que nos Latossolo e Podzólico a imobilização foi principalmente de origem microbiana. Nesses solos, entre 63 a 66\% (Latossolo) e 69 a $73 \%$ (Podzolico) do ${ }^{15} \mathrm{~N}$ imobilizado no solo foi encontrado na forma de $\mathrm{N}$ solúvel em ácido e não destilado (NSAnD).

No solo GPH houve pouca diferença entre os teores de ${ }^{15} \mathrm{~N}$ imobilizado nas frações NSAnD e N solúvel em ácido e destilado (NSAD). Entre 46 e $53 \%$ do ${ }^{15} \mathrm{~N}$ imobilizado ficou na fração NSAnD e entre 42 a $52 \%$ ficou na fração NSAD. O alto estoque de ${ }^{15} \mathrm{~N}$ imobilizado na fração NSAD pode estar relacionado com as características mineralógicas desse solo de várzea.

\section{BIBLIOGRAFIA CITADA}

Alfaia, S.S.; Nogueira, F.D. 1985. Estudo da capacidade de troca de cátions da fração mineral e orgânica de três solos da Amazônia Central. Ciência \& Prática, 9(1): 30-38.

Alfaia, S.S. 1997. Destino de adubos nitrogenados marcados com ${ }^{15} \mathrm{~N}$ em amostras de dois solos da Amazônia Central. Revista Brasileira de Ciência do Solo, 21(3): 379-385. 


\section{ACTA

Alfaia, S.S. 1995. Destino de fertilizantes nitrogenados marcados com ${ }^{15} \mathrm{~N}$ em um solo de várzea da Amazônia Central. In: Reunião Anual da Sociedade Brasileira para o Progresso da Ciência, $47^{a}$, São Luís, Anais, p 3.

Bremner, J.M. 1965. Inorganic forms of nitrogen. In: Black, C.A. (ed). Methods of soil analysis. Part 2. American Society of Agronomy, Madison, p.1179-1237.

Broadbent, F.E.; Taylor, K.B. 1962. Laboratory and greenhouse investigations of nitrogen immobilization. Soil Science Society American Proceedings, 26: 459-462.

Egoumenides, C.; Risterucci, A.; Melebou, K.E. 1987. Appréciation de la fertilisation azotée des sols tropicaux: études des fractions organiques de l'azote. L'Agronomie Tropical, 42(2): 85-93.

Embrapa. Centro Nacional de Pesquisa de solo. 1997. Manual de métodos de análise de solo. Vol. 2. ed. ver. atual, 212 pp. Rio de Janeiro, RJ.

Fiedler, R.; Proksch, G. 1975. The determination of nitrogen 15 by emission and mass spectrometry in biochemical analysis: a review. Analytica Chimica Acta, 78:1-62.

Giddens, J.; Hauck, R.D.; Adans, W.E.; Dawson, R.N. 1971. Forms of nitrogen in plant and soil sample. Soil Science Society American Journal, 63: 458-460.

Khan, S.U. 1971. Nitrogen fraction in gray wooded soil as influenced by long-term cropping systems and fertilizers. Canadian Journal Soil Science, 51: 431-437.

Jacquin, F. ; Cheloufi, H. ; Vong, P.C. 1992. Immobilization and mineralization kinetics of a nitrogen fertilizer in calcareous clayey soil (rendzina). The Science Total Environment, 117/118: 271278.
Jacquin, F.; Vong, P.C. 1989. Incorporation of a nitrogen fertilizer in the humified compounds of a typic hapludalf. The Science Total Environment, 81/82: 465-469.

Kelley, K.R.; Stevenson, F.J. 1995. Forms and nature of organic N in soil. Fertilizer Research, 42:1-11.

Stewart, B.A.; Porter, L.K.; Johnson, D.D. 1963. Immobilisation and mineralisation of nitrogen in several organic fraction of soil. Soil Science Society American Proceedings, 27: 302-304.

Schnitzer, M. 1981. Toward a better understanding of the interrelationship between Humus and nitrogen; a key economic and scientific problem in international agriculture. In: Colloque Humus et Azote, 1981, Anais, Reims-France, p.1-15.

Sulçe, S.; Palma-Lopez, D.; Jacquin, F.; Vong, P.C., Guiraud, G. 1996. Study of immobilization and remobilization of nitrogen fertilizer in cultivated soils by hydrolytic fraction. European Journal of Soil Science, 47(2): 249-255.

Vong, P.C. 1987. Contribution à l'étude cinétique des différents compartiments azotés contenus dans les sols cultivés après apports de fertilisants minéraux et organiques. Tese de Doutorado, École Nationale Supérieure d'Agrinomie et des Industries Alimentaire/ Institut National Polytechnique de Lorraine, Nancy, França. 217pp.

Vong, P.C.; Kabibou, I.; Jacquin, F. 1990. Etudes des correlations entre biomasse microbienne et differentes fractions d'azote organique presentes dans deux sols lorrains. Soil Biology Biochemistry, 22(3): 385-392.

Recebido em 26/05/2003

Aceito em 21/02/2006 